\title{
Selenium, selenoproteins and selenometabolites in mothers and babies at the time of birth
}

\author{
Cristina Santos ${ }^{1} \dagger$, Eduardo García-Fuentes ${ }^{2,3 *}$, Belén Callejón-Leblic ${ }^{4}$, Tamara García-Barrera ${ }^{4,5}$, \\ José Luis Gómez-Ariza ${ }^{4,5}$, Margaret P. Rayman ${ }^{6}$ and Inés Velasco ${ }^{5,7 *}+$ \\ ${ }^{1}$ Laboratory of Clinical Analysis, Hospital de Riotinto, Avda La Esquila 5; 21.660-Minas de Riotinto, Huelva, Spain \\ ${ }^{2}$ Digestive Unit, Institute of Biomedical Investigation of Málaga (IBIMA), Virgen de la Victoria University Hospital, Plaza del \\ Hospital Civil s/n, 29.009-Málaga, Spain \\ ${ }^{3}$ Biomedical Research Networking Centers in Physiology of Obesity and Nutrition (CIBEROBN), Plaza del Hospital Civil s/n, \\ 29.009-Málaga, Spain \\ ${ }^{4}$ Department of Chemistry and Materials Science, Faculty of Experimental Science, University of Huelva, Avda Fuerzas \\ Armadas s/n, 21071-Huelva, Spain \\ ${ }^{5}$ Research Center of Health and Environment (CYSMA), University of Huelva, Campus de El Carmen, Avda Fuerzas Armadas \\ $s / n, 21071-H u e l v a$, Spain \\ ${ }^{6}$ Department of Nutritional Sciences, Faculty of Health and Medical Sciences, University of Surrey, Guildford GU2 7XH, UK \\ ${ }^{7}$ Pediatrics, Obstetrics \& Gynecology Unit, Hospital de Riotinto, Avda La Esquila 5; 21.660-Minas de Riotinto, Huelva, Spain
}

(Submitted 6 February 2017 - Final revision received 30 March 2017 - Accepted 10 April 2017- First published online 23 May 2017)

\section{Abstract}

The deficiency of Se, an essential micronutrient, has been implicated in adverse pregnancy outcomes. Our study was designed to determine total serum Se, selenoproteins (extracellular glutathione peroxidase (GPx-3), selenoprotein P (SeP)), selenoalbumin (SeAlb) and selenometabolites in healthy women and their newborns at delivery. This cross-sectional study included eighty-three healthy mother-baby couples. Total Se and Se species concentrations were measured in maternal and umbilical cord sera by an in-series coupling of twodimensional size-exclusion and affinity HPLC. Additional measurements of serum SeP concentration and of serum GPx-3 enzyme activity were carried out using ELISA. Total Se concentration was significantly higher in maternal serum than in cord serum (68.9 (sD 15.2 ) and $56 \cdot 1$ (sD 14.6) $\mu \mathrm{g} / \mathrm{l}$, respectively; $P<0 \cdot 01$ ). There were significant correlations between selenoprotein and SeAlb concentrations in mothers and newborns, although they also showed significant differences in GPx-3 (11.2 (sD 3.7) $v .10 .5$ (sD 3.5) $\mu \mathrm{g} / 1 ; P<0.01$ ), SeP (42.5 (sD 9.5) $v .28 .1$ $(\mathrm{sD} 7.7) \mu \mathrm{g} / \mathrm{l} ; P<0.01$ ) and SeAlb (11.6 (sD 3.6) $v .14 .1$ (sD 4.3) $\mu \mathrm{g} / 1 ; P<0.01$ ) concentrations in maternal and cord sera, respectively. Serum GPx-3 activity and concentration were positively correlated in mothers $(r 0.33 ; P=0.038)$ but not in newborns. GPx-3 activity in cord serum was significantly correlated with gestational age $(r 0.44 ; P=0.009)$. SeAlb concentration was significantly higher in babies, whereas SeP and GPx-3 concentrations were significantly higher in mothers. The differences cannot be explained by simple diffusion; specific transfer mechanisms are probably involved. GPx-3 concentrations in mothers, at delivery, are related to maternal Se status, whereas the GPx-3 activity in cord serum depends on gestational age.

Key words: Selenium: Selenoproteins: Selenoprotein P: Gestation

Se is an essential micronutrient with a wide range of protective functions, which are exerted through its incorporation into selenoproteins ${ }^{(1)}$. Inadequate dietary Se intake can compromise human reproduction as Se deficiency has been associated with obstetric and perinatal complications such as male and female infertility, miscarriage, pre-eclampsia, intra-uterine growth restriction, preterm delivery and neural defects in the offspring $^{(2-4)}$.
The maternal-fetal transfer mechanisms for Se and selenoproteins have been studied in mice ${ }^{(5)}$ and in placental models in vitro $^{(6)}$, leading to the identification of some specific placental transporters ${ }^{(6,7)}$. Though a number of studies have measured Se concentrations in mothers and newborns at the time of birth, they have had contradictory results ${ }^{(8-10)}$. To some extent, these contradictions might be explained by differences in Se status between geographic regions ${ }^{(11)}$, distribution

Abbreviations: 2D/SE-AF-HPLC, two-dimensional size-exclusion and affinity HPLC; GPx-3, extracellular glutathione peroxidase; SeAlb, selenoalbumin; SeMetab, selenometabolites; SeP, selenoprotein P.

* Corresponding authors: E. García-Fuentes, email edugf1@gmail.com; Dr I. Velasco, fax +34959025 347, email inesvelas@msn.com

$\dagger$ These authors contributed equally to this work. 
patterns between Se species and by the reliability of the method of determination of $\mathrm{Se}^{(12)}$. Whatever the case, those studies were limited by measurement of only total serum/plasma Se, which does not allow distinction between different species of Se that may be transferred from the mother to the baby by different mechanisms.

In investigating the relative concentrations of Se in mothers and newborns it is important to take account of the different Se components in serum/plasma ${ }^{(13)}$ : two selenoproteins (selenoprotein $\mathrm{P}(\mathrm{SeP})^{(14)}$ and the extracellular glutathione peroxidase $(G P x-3))$, Se incorporated non-specifically as selenomethionine (SeMet) in lieu of methionine in albumin (selenoalbumin (SeAlb)) and other plasma proteins, and a small amount of non-proteinbound low molecular weight selenometabolites (SeMetab). SeP is the most abundant selenoprotein in plasma and is a good indicator of Se status in non-Se-replete humans ${ }^{(15)}$. The activity of GPX-3 in human serum is a complementary marker of Se status. Importantly, GPX-3 has been identified as a key enzyme in the defence against oxidative stress during decidualisation (the postovulatory process of endometrial remodelling in preparation for implantation) by reducing $\mathrm{H}_{2} \mathrm{O}_{2}$ in the endometrium ${ }^{(16)}$.

We have been able to determine these species in the serum from mother-baby pairs at the time of birth owing to our previous development of a two-dimensional chromatographic method for simultaneous speciation of serum GPX-3, SeP, SeAlb and SeMetab ${ }^{(17)}$. Although the accuracy of this method has been assessed by analysing a commercial human serum (BCR-637), it has not previously been applied in a clinical setting.

The aims of the current study were (i) to simultaneously determine Se, selenoproteins, SeAlb and SeMetab concentrations by this new method in both maternal and cord sera from uncomplicated pregnancies at the time of birth; (ii) to compare maternal and neonatal concentrations in order to elucidate potential maternal-fetal transfer mechanisms; (iii) to assess whether SeP measured by two-dimensional size-exclusion and affinity HPLC (2D/SE-AF-HPLC) corresponded to that measured by ELISA and (iv) to assess the coherence between GPx-3 measured both as a concentration and as an enzyme activity. Our study may also help to assess Se requirements in pregnancy, as recommended by the European Food Safety Authority Panel ${ }^{(18)}$

\section{Methods}

A cross-sectional study was performed on eighty-three healthy mother-baby couples. Participants were recruited at random from pregnant women without any maternal or neonatal risk factors, who gave birth at term, at the Department of Obstetrics and Gynecology, Hospital de Riotinto, during the years 2014-2015. Women with diseases, multiple gestation and with perinatal complications such as obstructed labour, low Apgar score (below 5) or cases where there was suspicion of infant pathology were excluded.

A full history was taken from the enrolled mothers, which included whether or not they took a multivitamin/mineral supplement (a standard formula approved by the Department of Health, containing $200 \mu \mathrm{g}$ iodide $\left(\mathrm{I}^{-}\right), 55 \mu \mathrm{g}$ Se, as sodium selenite, $10 \mathrm{mg} \mathrm{Zn}$ and $200 \mathrm{mg}$ DHA) during pregnancy. Medical data from clinical examination of mothers and babies were also recorded at birth.

This study was approved by the local ethics committee and written informed consent was obtained from all the participants. As we also collected samples from cord blood, the parents provided separate, signed, informed consent for this specific purpose.

\section{Analytical procedures}

Blood samples were collected from mothers during the $24 \mathrm{~h}$ before delivery. At birth, a sample of cord blood was obtained and its $\mathrm{pH}$ was measured. Serum was separated and frozen at $-80^{\circ} \mathrm{C}$ until further analysis.

Se and selenoprotein concentrations were measured by inseries 2D/SE-AF-HPLC, as previously described; this method overcomes common spectral interferences from chloride and bromide ${ }^{(17)}$. In brief, plasma and serum samples were filtered before injecting on to the chromatographic platform, which connected two in-series, stacked, $5 \mathrm{ml}$ HiTrap $^{\circledR}$ Desalting columns (for size exclusion separation; HiTrap-DesaltingSephadex G-25 Superfine; GE Health Bio-Science AB) and a dual-affinity column arrangement comprising a $1 \mathrm{ml}$ heparinsepharose column (HEP-HP; able to retain selectively SeP; GE Health Bio-Science AB) and a $1 \mathrm{ml}$ blue-sepharose column (BLU-HP; that retains both SeP and SeAlb; GE Health Bio-Science AB) interconnected by a six-port column-switching valve, which were coupled in series to an inductively coupled plasma MS system (ICP-MS Agilent 7500ce; Agilent Technologies) by means of a $30-\mathrm{cm}$ polyether ether ketone (PEEK) tubing $(0.6 \mathrm{~mm}$ i.d.) for Se detection. Ammonium acetate $0.05 \mathrm{M}$ (at $\mathrm{pH} 7.5$ ), phase A and $1.5 \mathrm{~m}$ phase B were successively used as the mobile phase. Postcolumn isotope dilution analysis was performed by the introduction of ${ }^{74} \mathrm{Se}$ via a T-connector.

Chromatographic performance was checked regularly by measuring control standards to ensure enough separation between species and to ensure method sensitivity after a considerable number of analyses. The proposed speciation method has been validated using a CRM of human serum (BCR-637) certified for total Se content (Se total $=81(\mathrm{sD} 7) \mathrm{ng} / \mathrm{ml})$. The concentration of different Se species in BCR-637 obtained with this method has been compared with previous results for this CRM from Jitaru et al. ${ }^{(19)}$. The relative standard deviation (RSD, \%) and the detection limits (DL, ng/g of Se) are as follows: GPx-3, RSD 21 and DL 0.2; SeP, RSD 12 and DL 0.7; SeAlb, RSD 9 and DL 0.9.

SeP concentration was also analysed by ELISA (USCN Business Co., Ltd), and GPx-3 activity was analysed by a commercial kit (Cayman Chemical).

\section{Statistical analysis}

Sample size calculation: with thirty-eight subjects per motherbaby group, and a SD of $15 \mu \mathrm{g} / \mathrm{l}$ of Se in mean serum Se concentration of $71 \mu \mathrm{g} / \mathrm{l}$ (obtained from a recent study of Spanish pregnant women $(71.2 \text { (SD 14.9)) })^{(20)}$, our study had $80 \%$ power to detect a difference of at least $10 \mu \mathrm{g} / \mathrm{l}$ in total serum Se in mother-baby groups at a two-sided, $5 \%$ significance level. We included eighty-three healthy mother-baby couples.

As our sample included women who took a multivitamin/ mineral supplement and women who did not, a comparison of 
means between these two groups was made to see whether this supplementation had a significant effect. Assuming a two-sided hypothesis, the conventional $\alpha$ and $\beta$ levels, and a proportion of 3:1 for the studied groups (the use of multivitamin/mineral supplements in our population barely reached a quarter of pregnant women), the sample size calculated was nineteen women for the supplemented group and fifty-seven pregnant women for the non-supplemented group. We included twenty-one and sixty-two women, respectively.

Data are presented as medians and standard deviations for continuous variables and as percentages for categorical variables. The contrast hypothesis for two samples was evaluated using Fisher's exact test for categorised variables and Student's $t$ test for continuous variables. The Wilcoxon signed-rank test was used to compare matched samples. The correlation between variables was determined using the Spearman test, designing multiple linear regression models in those cases where it was desired to predict the variance adjusted for other variables, besides the main variable. The contrast hypothesis for more than two samples was determined with an ANOVA. All $P$ values were two-sided, and statistical significance was declared at $P<0 \cdot 05$. All data were analysed using SPSS 20.0 (IBM SPSS Statistics).

\section{Results}

\section{Maternal and neonatal characteristics}

Table 1 shows maternal and neonatal characteristics. As exclusively healthy pregnant women were recruited, the caesarean section rate was only $7.5 \%$ (compared with the usual caesarean section rate at our centre of about $16 \%$ ) and perinatal outcomes were good (there were no admissions to the Neonatal Intensive Care Unit).

No correlations were found between maternal age, parity, BMI or level of education and maternal Se concentration, nor were any correlations found between neonatal weight at birth or gestational age with maternal or neonatal serum Se concentrations. However, there was a negative correlation between hours of fasting before blood drawing and total Se concentration in maternal serum $(r-0.30 ; P<0.05)$.

Women who did not take multivitamin/mineral supplements (containing $55 \mu \mathrm{g} \mathrm{Se}$ ) were significantly younger than women who did take them (29.42 (SD 5.35) v. 31.16 (SD 4.80) years, respectively; $P<0.05$ ). No significant differences in parity, BMI or level of education were found between women who took multivitamin/mineral supplements and those who did not take them (data not shown).

\section{Selenium and selenoprotein concentrations by two-dimensional size-exclusion and affinity HPLC}

Total Se and selenoprotein concentrations in maternal and cord sera are summarised in Table 2. Total Se, GPx-3 and SeP concentrations were significantly higher in maternal serum than in cord serum, whereas SeAlb was significantly more concentrated in cord serum. There was no significant difference in the SeMetab concentration between maternal and cord sera.
Table 1. Main characteristics of participants (Mean values and standard deviations)

\begin{tabular}{|c|c|c|}
\hline & Mean & SD \\
\hline \multicolumn{3}{|l|}{ Maternal characteristics } \\
\hline Maternal age (years) & $29 \cdot 7$ & $5 \cdot 7$ \\
\hline Maternal weight (kg) & $81 \cdot 48$ & $14 \cdot 35$ \\
\hline $\mathrm{BMI}\left(\mathrm{kg} / \mathrm{m}^{2}\right)$ & 31.84 & 8.32 \\
\hline \multicolumn{3}{|l|}{ Parity (\%) } \\
\hline Primiparous & $49 \cdot 4$ & \\
\hline Multiparous & $51 \cdot 6$ & \\
\hline \multicolumn{3}{|l|}{ Previous miscarriages (\%) } \\
\hline Yes & $22 \cdot 2$ & \\
\hline No & $77 \cdot 8$ & \\
\hline \multicolumn{3}{|l|}{ Use of supplements } \\
\hline None & 11/83 (13.3\%) & \\
\hline Potassium iodide & $51 / 83(61.4 \%)$ & \\
\hline Multivitamins & $21 / 83(25.3 \%)$ & \\
\hline \multicolumn{3}{|l|}{ Level of education (\%) } \\
\hline Low & 90 & \\
\hline Medium & 10 & \\
\hline High & 0 & \\
\hline \multicolumn{3}{|l|}{ Working woman (\%) } \\
\hline No & 80 & \\
\hline Yes & 20 & \\
\hline \multicolumn{3}{|l|}{ Consumption of iodised salt (\%) } \\
\hline No & $69 \cdot 5$ & \\
\hline Yes & $30 \cdot 5$ & \\
\hline Weight gain during pregnancy $(\mathrm{kg})$ & $12 \cdot 64$ & 5.06 \\
\hline \multicolumn{3}{|l|}{ Type of delivery (\%) } \\
\hline Normal vaginal delivery & 75 & \\
\hline Vacuum/forceps & $17 \cdot 5$ & \\
\hline Caesarean section & 7.5 & \\
\hline \multicolumn{3}{|l|}{ Neonatal characteristics } \\
\hline Gestational age at birth (d) & $275 \cdot 2$ & $8 \cdot 4$ \\
\hline Birth weight (g) & $3392 \cdot 84$ & $408 \cdot 91$ \\
\hline Birth height (cm) & $50 \cdot 32$ & 1.56 \\
\hline Head circumference $(\mathrm{cm})$ & 33.48 & 1.44 \\
\hline Abdominal circumference $(\mathrm{cm})$ & $33 \cdot 40$ & 1.67 \\
\hline
\end{tabular}

The chromatograms of maternal and umbilical cord sera are shown in Fig. 1.

The correlations between maternal and cord Se species were significant in all cases, reaching the highest value for GPx-3 and the lowest for SeP.

The proportion of Se species in maternal and cord sera was different; whereas SeP accounted for $65 \%$ of total Se in maternal serum, it only represented $50 \%$ of total Se in cord serum. By contrast, SeAlb constituted $15 \%$ of total Se in maternal serum but $28 \%$ in cord serum. The percentages for GPx-3 and SeMetab were similar in maternal and cord sera: $14 \%$ for GPX-3 in both, and 7 and $8 \%$ for SeMetab in maternal and cord sera, respectively (Fig. 2(a)). When the Se species concentrations are represented graphically, the different pattern in maternal and cord sera is apparent (Fig. 2(b)).

There were no significant differences in the concentrations of Se species in maternal or cord sera between women who took multivitamins/minerals containing se and those who did not (Table 3).

Maternal total serum Se was strongly correlated with all the Se species in maternal serum, that is, GPx-3 $(r 0.71 ; P<0 \cdot 1)$, SeP $(r 0.89 ; P<0.01)$, SeAlb $(r 0.80 ; P<0.01)$ and SeMetab $(r 0.42$; $P<0.01)$, and moderately correlated with the concentrations of the Se species in cord serum, that is, total Se $(r 0.56 ; P<0.01)$, 
Table 2. Comparisons between concentrations of total selenium, selenoproteins, selenoalbumin (SeAlb) and selenometabolites (SeMetab) in maternal and cord sera measured by two-dimensional size-exclusion and affinity HPLC (2D/SE-AF-HPLC) (Medians and standard deviations)

\begin{tabular}{|c|c|c|c|c|c|c|c|}
\hline & \multicolumn{2}{|c|}{ Maternal serum } & \multicolumn{2}{|c|}{ Cord serum } & \multicolumn{2}{|c|}{ Correlation } & \multirow[b]{2}{*}{ Difference in means $(P)$} \\
\hline & Median & SD & Median & SD & $r$ & $P$ & \\
\hline Total Se $(\mu \mathrm{g} / \mathrm{l})$ & 68.95 & $15 \cdot 23$ & $56 \cdot 14$ & 14.61 & 0.56 & $<0.01$ & $<0.01$ \\
\hline GPx-3 concentration $(\mu \mathrm{g} / \mathrm{l})$ & $11 \cdot 24$ & 3.68 & $10 \cdot 46$ & 3.48 & 0.74 & $<0.01$ & $<0.01$ \\
\hline SeP concentration $(\mu \mathrm{g} / \mathrm{l})$ & 42.49 & 9.49 & 28.06 & 7.69 & 0.25 & $<0.01$ & $<0.01$ \\
\hline SeAlb $(\mu \mathrm{g} / \mathrm{l})$ & 11.35 & 3.59 & $14 \cdot 14$ & $4 \cdot 26$ & 0.60 & $<0.01$ & $<0.01$ \\
\hline SeMetab $(\mu \mathrm{g} / \mathrm{l})$ & 4.32 & 2.86 & 3.98 & 2.38 & 0.60 & $<0.01$ & NS \\
\hline
\end{tabular}

GPx-3, extracellular glutathione peroxidase; SeP, selenoprotein P.
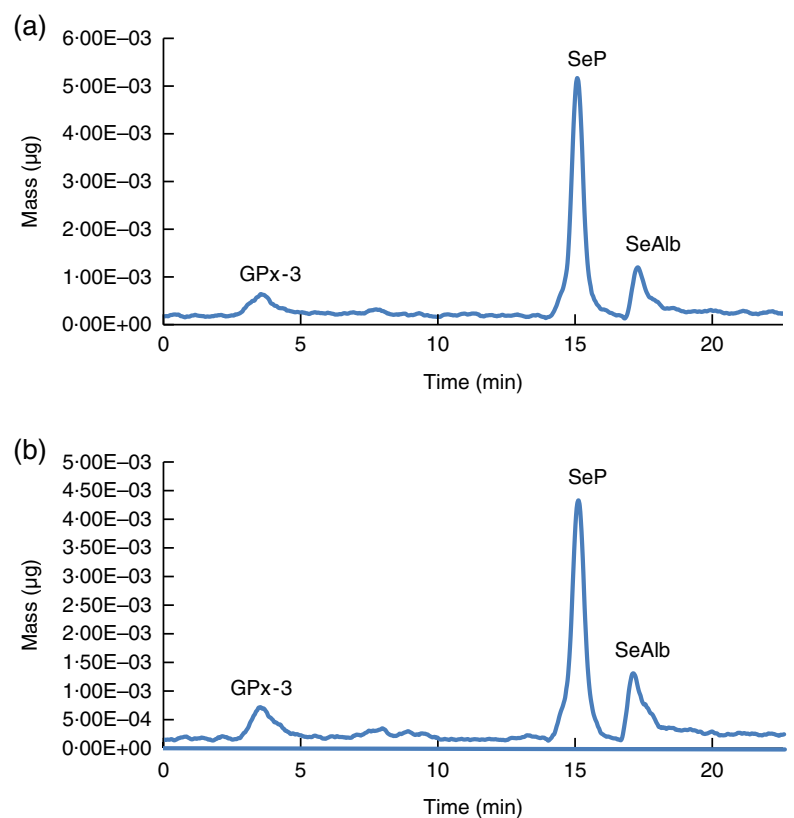

Fig. 1. Selenoprotein chromatograms in (a) maternal and (b) umbilical cord sera. GPx-3, extracellular glutathione peroxidase; SeP, selenoprotein P; SeAlb, selenoalbumin.

GPx-3 $(r 0 \cdot 47 ; P<0 \cdot 01)$, SeP $(r 0.43 ; P<0 \cdot 01)$, SeAlb $(r 0 \cdot 45$ $P<0.01)$ and SeMetab $(r 0.48 ; P<0.01)$

Total Se in cord serum was correlated with all the Se species studied in cord serum, that is, GPx-3 $(r 0.78 ; P<0.1)$, SeP $(r 0.89 ; P<0.01)$, SeAlb $(r 0 \cdot 80 ; P<0.01)$ and SeMetab $(r 0.60$; $P<0.01)$, and moderately correlated with the concentration of the Se species in maternal serum, that is, GPX-3 ( $r$ 0.64; $P<0.01)$, SeP $(r 0.32 ; P<0.01)$, SeAlb $(r 0.61 ; P<0.01)$ and SeMetab $(r 0.66 ; P<0.01)$.

Negative correlations were found between umbilical cord $\mathrm{pH}$ and maternal SeAlb $(r-0.33 ; P=0 \cdot 01)$, cord total Se $(r-0 \cdot 30$; $P=0 \cdot 04)$ and cord SeAlb $(r-0 \cdot 30 ; P=0 \cdot 02)$.

\section{Extracellular glutathione peroxidase activity}

GPx-3 activity was significantly higher in maternal serum than in cord serum (112.87 (sD 68.91) and 38.67 (sD 25.03) nmol/min per $\mathrm{ml}$, respectively; $P<0.01)$, and there was no correlation between the activities $(r 0 \cdot 12 ; P=0 \cdot 48)$. There was a significant (a)

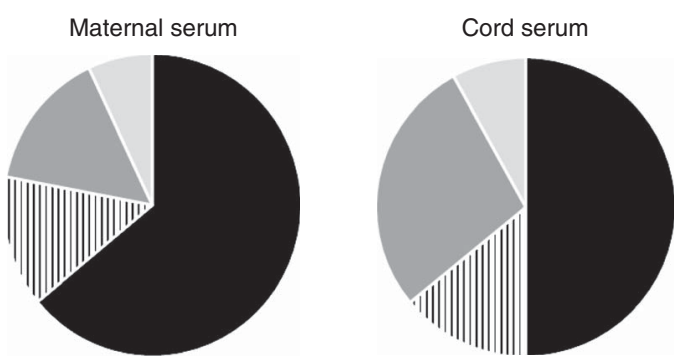

(b)

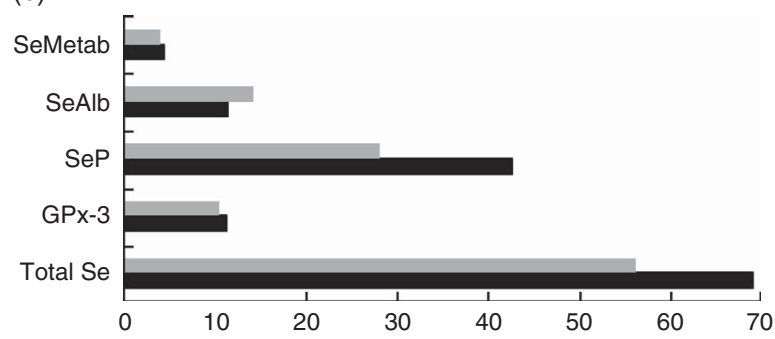

Fig. 2. Comparison between (a) percentage of selenoproteins and selenometabolites (SeMetab) in maternal and cord sera and (b) concentrations of total selenium, selenoproteins and SeMetab in maternal and cord sera $(\mu \mathrm{g} / \mathrm{l})$. (a): $\square$, selenoprotein $\mathrm{P}(\mathrm{SeP}) ; \boldsymbol{W}$, extracellular glutathione peroxidase (GPX-3); $\square$, selenoalbumin (SeAlb); $\square$, SeMetab. (b): $\square$, cord serum;, , maternal serum.

correlation between GPx-3 concentration and its activity in maternal serum $(r 0.33 ; P=0.04)$, but this correlation was not apparent in cord serum (data not shown). Maternal GPx-3 activity also correlated significantly with total Se concentration in maternal serum $(r 0.36 ; P=0.03)$ and with SeMetab concentration in cord serum $(r 0.50 ; P<0 \cdot 01)$, but not with other Se species in either maternal or cord sera (data not shown). GPx-3 activity in cord serum was significantly correlated with birth weight $(r 0.39 ; P=0.02)$ and gestational age $(r 0.43 ; P<0.01)$. This last correlation remained significant after adjusting for birth weight and height $\left(R^{2} 0.23 ; P=0.04\right)$. However, GPx-3 activity in cord serum did not correlate with total Se or Se species either in cord serum or maternal serum (data not shown).

\section{Selenoprotein P measured by ELISA}

SeP concentration determined by ELISA was significantly different in maternal and cord sera (38.27 (SD 12.34) and 1.93 (sD 0.98$) \mu \mathrm{g} / 1$, respectively; $P<0.01$ ). No correlation was found 
Table 3. Concentration of various selenium species in maternal and cord sera in women who took multivitamin/mineral supplement containing selenium during pregnancy and those who did not take such supplements (Medians and standard deviations)

\begin{tabular}{|c|c|c|c|c|c|}
\hline & \multicolumn{2}{|c|}{ No supplements ( $n$ 62) } & \multicolumn{2}{|c|}{ Multivitamins ( $n$ 21) } & \multirow[b]{2}{*}{$P$} \\
\hline & Median & SD & Median & SD & \\
\hline Maternal total Se $(\mu \mathrm{g} / \mathrm{l})$ & $67 \cdot 40$ & $14 \cdot 24$ & 69.72 & $15 \cdot 32$ & 0.54 \\
\hline Maternal GPx-3 $(\mu \mathrm{g} / \mathrm{l})$ & $10 \cdot 84$ & 3.38 & 11.57 & 3.92 & 0.43 \\
\hline Maternal SeP $(\mu \mathrm{g} / \mathrm{l})$ & 41.44 & 9.04 & $44 \cdot 86$ & $10 \cdot 25$ & 0.16 \\
\hline Maternal SeAlb $(\mu \mathrm{g} / \mathrm{l})$ & $11 \cdot 16$ & 3.38 & 11.09 & 3.31 & 0.41 \\
\hline Maternal SeMetab $(\mu \mathrm{g} / \mathrm{l})$ & 4.35 & 2.96 & $3 \cdot 21$ & 1.45 & 0.18 \\
\hline Cord total Se $(\mu \mathrm{g} / \mathrm{l})$ & $55 \cdot 24$ & $13 \cdot 32$ & $55 \cdot 12$ & 11.75 & 0.97 \\
\hline Cord GPx-3 $(\mu \mathrm{g} / \mathrm{l})$ & $10 \cdot 13$ & 3.24 & $10 \cdot 84$ & 3.52 & 0.42 \\
\hline Cord SeP $(\mu \mathrm{g} / \mathrm{l})$ & $28 \cdot 12$ & $7 \cdot 70$ & $27 \cdot 12$ & $6 \cdot 36$ & 0.62 \\
\hline Cord SeAlb $(\mu \mathrm{g} / \mathrm{l})$ & 13.49 & 3.46 & $14 \cdot 51$ & 3.62 & 0.28 \\
\hline Cord SeMetab $(\mu \mathrm{g} / \mathrm{l})$ & 3.82 & $2 \cdot 16$ & 3.67 & $2 \cdot 26$ & 0.83 \\
\hline
\end{tabular}

GPx-3, extracellular glutathione peroxidase; SeP, selenoprotein P; SeAlb, selenoalbumin; SeMetab, Selenium metabolites.

between SeP in maternal and cord sera when measured by ELISA. There was no correlation between SeP concentrations measured by HPLC and ELISA in either maternal serum or cord serum (data not shown). Although SeP concentration in maternal serum measured by HPLC and ELISA was not significantly different (42.49 (SD 9.49) and 38.27 (SD 12.34) $\mu \mathrm{g} / \mathrm{l}$, respectively; $P=0 \cdot 10$ ), there was a significant difference between the concentrations obtained by these two methods of determination in cord serum (28.06 (SD 7.69) $\mu \mathrm{g} / 1$ by HPLC and $1.93(\mathrm{sD} 0.98) \mu \mathrm{g} / \mathrm{l}$ by ELISA; $P<0.01$ ). Maternal SeP concentration measured by ELISA correlated significantly with GPX-3 concentration in cord serum $(r 0.33 ; P=0.04)$.

\section{Discussion}

This is the first study to simultaneously analyse total Se and Se species in maternal and cord sera at the time of birth using a new optimised HPLC method. Although some of these factors have previously been measured at delivery, the interrelationships between the different Se species in maternal and cord sera are largely unknown, as is the biological significance of such relationships. Our findings indicate that, at birth, total Se, GPx-3 and SeP concentrations are significantly higher in maternal serum than in cord serum, whereas the opposite is the case for SeAlb. In general, simple diffusion cannot explain the maternalneonatal transfer of Se and Se species about the time of birth.

Oxidative stress is tightly related to perinatal morbidity and mortality; therefore, the antioxidant selenoproteins may have a central role in protecting against adverse pregnancy outcomes ${ }^{(21)}$. Thus, total Se concentration and GPx-3 activity in cord serum were found to increase with advancing pregnancy ${ }^{(22)}$, whereas oxidative stress during labour was associated with elevated fetal GPx-3 activity ${ }^{(23)}$. A study in rats showed that a sufficient Se supply was required to ensure antioxidative protection to the fetus during the oxygen transformations that take place during delivery and early postnatal life ${ }^{(24)}$. By contrast, in a UK population of slightly higher Se status than ours, maternal blood Se concentrations fell from 12 to 35 weeks of gestation (believed to be partly due to the expansion of plasma volume and partly due to receptor-mediated transfer of SeP from mother to fetus) and maternal GPX-3 activity did not change ${ }^{(25)}$.
Our study found lower Se concentrations than those reported in other studies of Spanish pregnant women ${ }^{(20,26)}$. Using the most restrictive cut-off point of $70 \mu \mathrm{g} / \mathrm{l}$ of total serum Se concentration required to optimise GPx-3 activity ${ }^{(27)}$, fifty-seven of eighty-three (69\%) pregnant women in our study had insufficient serum Se to fulfil this requirement. However, based on alternative estimates of the range of serum Se concentration required for optimal GPx-3 activity, that is, 89-114 $\mu \mathrm{g} / \mathrm{l}^{(11)}, 90 \%$ of the women in our sample did not reach this optimal status.

The dietary intake of Se is the major determining factor for serum Se concentration. As we used data from medical records, information on dietary Se intake was not available. However, other factors have been reported to be associated with Se status such as sex ${ }^{(28,29)}$, age, $\mathrm{BMI}^{(28)}$, education ${ }^{(30)}$, socio-economic status $^{(28,30)}$ or physical activity ${ }^{(29)}$, as well as location ${ }^{(31)}$. In this regard, our patients came from a non-urban area in southern Spain where Se intake is traditionally low ${ }^{(31)}$. Furthermore, most of them were overweight or obese, had a low level of education and had never worked or were unemployed. All these factors lead us to speculate that they were unlikely to eat the traditional diet with high consumption of vegetables and cereals ${ }^{(32)}$.

It might have been expected that pregnant women who took multivitamins/multiminerals containing sodium selenite would have had significantly higher concentrations of at least some Se species than those who did not, as sodium selenite can be used for the synthesis of selenoproteins such as GPx-3 or SeP (though it will not increase SeAlb) ${ }^{(12)}$. However, the absence of higher concentrations in the women on supplements is not altogether surprising as the Se dose was relatively low $(55 \mu \mathrm{g} / \mathrm{d})$; only $25 \%$ of the women took such supplements and we have no data on their compliance. Furthermore, in vivo human studies showed that selenite is only moderately absorbed (between 56 and $85 \%)^{(33)}$.

In recent years, Se status in maternal and cord blood at delivery has been determined in a number of studies, though with varied outcomes ${ }^{(34-36)}$. Al-Saleh et al. ${ }^{(34)}$ examined the role of Se in reducing oxidative stress induced by $\mathrm{Cd}$ and its impact on birth measures. They concluded that the extent of benefit afforded by Se is not governed only by its concentration but also by the different chemical forms of Se that interact with various proteins. Chen et al. ${ }^{(35)}$ attempted to clarify 
concentrations for $\mathrm{Cd}, \mathrm{Hg}, \mathrm{Pb}$ and $\mathrm{Se}$ in mothers and newborns and their placental transfer. Finding a high degree of maternalfetal Se transfer, they concluded that there was free transplacental passage of Se from mother to fetus. However, our results do not support that conclusion; the different pattern of transfer of SeP and SeAlb implies that different mechanisms are involved in the transfer of these different Se species from mother to fetus.

It appears likely that receptor-mediated uptake exists in humans, as previously found in pregnant mice ${ }^{(5)}$. Burk et al. ${ }^{(5)}$ identified two mechanisms of Se transfer; from early to midgestation, plasma GPx-3 and SeP were transported via the uterine fluid, probably by pinocytosis, whereas in the latter half of gestation, placental transfer of maternal SeP occurred through the apoE receptor 2. In fact, the higher levels of SeAlb in cord serum compared with the levels in maternal serum suggest the existence of a further maternal-fetal Se transfer mechanism ${ }^{(5,37)}$. Studies in mice and rats suggest that this other pathway involves the transfer of Se as SeMet-containing proteins or SeMet itself (via methionine transporters) to the fetus ${ }^{(5,37)}$. This mechanism depends on the amount of SeMet in the diet of the mother, appears to be unregulated and is highly dependent on the mother's Se status; thus, it will be less effective under conditions of Se deficiency ${ }^{(5)}$. The similar concentrations for SeMetab and their strong correlation in maternal and cord sera can be explained by a simple diffusion mechanism of trans-placental passage that does not occur for other Se species.

GPx-3 activity was measured to see whether there was a correlation between GPx-3 concentrations and GPX-3 activity. As expected, the GPx-3 activity in maternal serum significantly correlated with maternal total Se and GPx-3 concentrations, but GPx-3 activity in cord serum only correlated with gestational age and birth weight. These results are in agreement with previous findings ${ }^{(38)}$; it seems that GPx-3 activity in mothers at delivery is related to maternal Se status, whereas the GPx-3 activity in cord serum depends on gestational age ${ }^{(22)}$.

Finally, our study compared different methods of determining SeP: 2D/SE-AF-HPLC and ELISA. The growing replacement of traditional immunoassay techniques by liquid chromatography tandem MS has highlighted the advantages and limitations of each system as well as the disparity in results by the two methods ${ }^{(39)}$. These two methods have been previously used and published ${ }^{(17,40)}$. In our sample, the concentration of SeP in maternal serum measured by both methods gave a similar result, but this was not the case in cord serum where SeP concentration measured by ELISA was significantly lower than that measured by HPLC, and did not correlate with any other parameters. Although these results may suggest that ELISA is not a reliable method of quantifying SeP in cord serum, other explanations are possible. SeP has three transcripts in humans, although all variants begin with the same non-coding exon ${ }^{(41)}$. The most abundant transcript in all tissues was SePa. However, all variants ( $\mathrm{SePa}, \mathrm{b}$ and c) showed tissue and developmental stage-specific expression patterns in neonatal and adult tissues $^{(41)}$. These transcripts can synthesise different isoforms, varying in size and Se content ${ }^{(41)}$, as previously proposed ${ }^{(42)}$. Moreover, the silencing of each transcript results in a differential synthesis of SeP isoforms ${ }^{(41)}$. In this respect, the antibodies used in ELISA kits could have a different affinity for the SeP isoforms present in serum. This could affect the binding of the antibody to the SeP protein in the ELISA and therefore give different results than techniques that do not use antibodies. A further determination of SeP levels by the use of kits from other suppliers could be considered but, unfortunately, we have no remaining cord blood samples. Alternatively, this discordance in the results of SeP concentration between 2D/SE-AF-HPLC and ELISA may suggest that, in the newborn, the predominant SeP isoform is different from that found in the mothers and, perhaps, with different functions ${ }^{(41)}$. It is also likely that full-length SeP has a higher Se content than the shorter isoforms ${ }^{(15)}$. It has been suggested that while the liver exports full-length $\mathrm{SeP}$, other tissues secrete mostly shorter SeP isoforms, possibly for local use as a redox enzyme or as a signalling molecule ${ }^{(15)}$. Our finding supports the hypothesis that circulating truncated $\mathrm{SeP}$ isoforms might not reach the concentrations necessary for successful identification by ELISA ${ }^{(43)}$.

The current study has some limitations. First, the absence of a FFQ did not allow us to assess Se intake. Furthermore, the small proportion ( $25 \%$ ) of women taking a supplement containing Se and the lack of compliance data may have prevented us from finding significantly higher markers of status in that group.

Our study has also certain strengths; our design made possible the collection of data and samples from healthy pregnant women and their newborns at the time of birth. The multiple biomarkers studied from the same patients and the availability of a robust and reliable method to simultaneously determine different Se species gave us an insight into the relationships between these Se species in mothers and newborns.

\section{Conclusion}

There is a different pattern of Se species found in maternal and cord sera at the time of birth, which suggests the existence of different mechanisms of trans-placental passage of Se species from mother to fetus, which are species dependent.

The lack of concordance in the SeP concentration measured by 2D/SE-AF-HPLC and ELISA suggests the possibility that the predominant $\mathrm{SeP}$ isoform in the newborn may be different from that found in the mother; this possibility needs to be followed up in a later study.

\section{Acknowledgements}

The authors sincerely thank all the mothers and their babies for voluntarily participating in the study and the staff of the Maternity ward for their involvement and support.

This study was undertaken with the aid of a grant from the Consejería de Salud of the Junta de Andalucía, Spain (PI-0373/ 2012) and by projects CTM2012-38720-C03-01 from the Spanish Ministry of Economy and Competitiveness (MINECO) and P12FQM-0442 from the Regional Ministry of Economy, Innovation, Science and Employment (Andalusian Government, Spain). B. C.-L. thanks the Spanish Ministry of Education, Culture and Sport for a doctoral grant.

C. S. and I. V. designed the study, recruited participants, collected the samples and wrote the manuscript. E. G.-F. performed the biochemical tests and the statistical analysis. 
T. G.-B. and J. L. G.-A. designed the analytical experiments, the instrumental couplings and supervised the results. C. S. and B. C.-L. performed the analytical experiments and the tandem MS analysis. M. P. R. contributed intellectually to the study and to the writing of the manuscript.

The authors declare that there are no conflicts of interest.

\section{References}

1. Duntas LH \& Benvenga S (2015) Selenium: an element for life. Endocrine 48, 756-775.

2. Pieczyńska J \& Grajeta H (2015) The role of selenium in human conception and pregnancy. J Trace Elem Med Biol 29, 31-38.

3. Rayman MP, Bode P \& Redman CW (2003) Low selenium status is associated with the occurrence of the pregnancy disease preeclampsia in women from the United Kingdom. Am J Obstet Gynecol 189, 1343-1349.

4. Rayman MP, Wijnen H, Vader H, et al. (2011) Maternal selenium status during early gestation and risk for preterm birth. Can Med Assoc J 183, 549-555.

5. Burk RF, Olson GE, Hill KE, et al. (2013) Maternal-fetal transfer of selenium in the mouse. FASEB J 27, 3249-3256.

6. Nandakumaran M, Dashti HM, Al-Saleh E, et al. (2003) Transport kinetics of zinc, copper, selenium, and iron in perfused human placental lobule in vitro. Mol Cell Biochem 252, 91-96.

7. Miyauchi S, Srinivas SR, Fei YJ, et al. (2006) Functional characteristics of NaS2, a placenta-specific $\mathrm{Na}^{+}$-coupled transporter for sulfate and oxyanions of the micronutrients selenium and chromium. Placenta 27, 550-559.

8. Jariwala M, Suvarna S, Kiran Kumar G, et al. (2014) Study of the concentration of trace elements $\mathrm{Fe}, \mathrm{Zn}, \mathrm{Cu}$, Se and their correlation in maternal serum, cord serum and colostrums. Indian J Clin Biochem 29, 181-188.

9. Bermúdez L, García-Vicent C, López J, et al. (2015) Assessment of ten trace elements in umbilical cord blood and maternal blood: association with birth weight. J Transl Med 13, 291.

10. Özdemir HS, Karadas F, Pappas AC, et al. (2008) The selenium levels of mothers and their neonates using hair, breast milk, meconium, and maternal and umbilical cord blood in Van Basin. Biol Trace Elem Res 122, 206-215.

11. Rayman MP. (2012) Selenium and human health. Lancet 379 , 1256-1268.

12. Fairweather-Tait SJ, Collings R \& Hurst R (2010) Selenium bioavailability : current knowledge and future research. $A m \mathrm{~J}$ Clin Nutr 91, 1484S-1491S.

13. Combs GF, Watts JC, Jackson MI, et al. (2011) Determinants of selenium status in healthy adults. Nutr J 10, 75.

14. Gladyshev VN, Arnér ES, Berry MJ, et al. (2016) Selenoprotein gene nomenclature. J Biol Chem 291, 24036-24040.

15. Burk RF \& Hill KE (2009) Selenoprotein P - expression, functions, and roles in mammals. Biochim Biophys Acta 1790, $1441-1447$.

16. Xu X, Leng JY, Gao F, et al. (2014) Differential expression and anti-oxidant function of glutathione peroxidase 3 in mouse uterus during decidualization. FEBS Lett 588, 1580-1589.

17. García-Sevillano MA, García-Barrera T \& Gómez-Ariza JL (2013) Development of a new column switching method for simultaneous speciation of selenometabolites and selenoproteins in human serum. J Chromatogr A 1318, 171-179.

18. European Food Safety Authority (EFSA) Panel on Dietetic Products, Nutrition and Allergies (NDA) (2014) Scientific opinion on dietary reference values for selenium. EFSA J 12, 3846.

19. Jitaru P, Roman M, Barbante C, et al. (2010) Challenges in the accurate speciation analysis of selenium in humans: first report on indicative levels of selenoproteins in a serum certified reference material for total selenium (BCR-637). Accred Qual Assur 15, 343-350.

20. Bermúdez L, García-Vicent C, López J, et al. (2015) Assessment of ten trace elements in umbilical cord and maternal blood: association with birth weight. J Transl Med 13, 291.

21. Mariath AB, Bergamaschi DP, Rondó PHC, et al. (2011) The possible role of selenium status in adverse pregnancy outcomes. Br J Nutr 105, 1418-1428.

22. Makhoul IR, Sammour RN, Diamond E, et al. (2004) Selenium concentrations in maternal and umbilical cord blood at 24-42 weeks of gestation: basis for optimization of selenium supplementation to premature infants. Clin Nutr 23, 373-381.

23. Katzer D, Mueller A, Welzing L, et al. (2015) Antioxidative status and oxidative stress in the fetal circulation at birth: the effects of time of delivery and presence of labor. Early Hum Dev 91, 119-124.

24. Nogales F, Ojeda ML, Fenutría M, et al. (2013) Role of selenium and glutathione peroxidase on development, growth, and oxidative balance in rat offspring. Reproduction 146, 659-667.

25. Rayman MP, Bath SC, Westaway J, et al. (2015) Selenium status in UK pregnant women and its relationship with hypertensive conditions of pregnancy. Br J Nutr 113, 249-258.

26. Izquierdo Alvarez S, Castañón SG, et al. (2007) Updating of normal levels of copper, zinc and selenium in serum of pregnant women. J Trace Elem Med Biol 21, 49-52.

27. Combs GF Jr (2015) Biomarkers of selenium status. Nutrients 7, 2209-2236.

28. Méplan C, Crosley LK, Nicol F, et al. (2007) Genetic polymorphisms in the human selenoprotein $\mathrm{P}$ gene determine the response of selenoprotein markers to selenium supplementation in a gender-specific manner (the SELGEN study). FASEB J 21, 3063-3074.

29. Millán-Adame E, Florea D, Sáez Pérez L, et al. (2012) Deficient selenium status of a healthy adult Spanish population. Nutr Hosp 27, 524-528.

30. Stranges S, Laclaustra M, Ji C, et al. (2010) Higher selenium status is associated with adverse blood lipid profile in British adults. J Nutr 140, 81-87.

31. Matos-Reyes MN, Cervera ML, Campos RC, et al. (2010) Total content of As, Sb, Se, Te and Bi in Spanish vegetables, cereals and pulses and estimation of the contribution of these foods to the Mediterranean daily intake of trace elements. Food Chem 122, 188-194.

32. Sánchez C, López-Jurado M, Aranda P, et al. (2010) Plasma levels of copper, manganese and selenium in an adult population in southern Spain: influence of age, obesity and lifestyle factors. Sci Total Environ 408, 1014-1020.

33. Moreda-Piñeiro J, Moreda-Piñeiro JA \& Bermejo-Barrera P (2017) In vivo and in vitro testing for selenium and selenium compounds bioavailability assessment in foodstuff. Crit Rev Food Sci Nutr 57, 805-833.

34. Al-Saleh I, Al-Rouqi R, Angela C, et al. (2015) Interaction between cadmium (Cd), selenium (Se) and oxidative stress biomarkers in healthy mothers and its impact on birth anthropometric measures. Int J Hyg Environ Health 218, 66-90.

35. Chen Z, Myers R, Wei T, et al. (2014) Placental transfer and concentrations of cadmium, mercury, lead, and selenium in mothers, newborns, and young children. J Expo Sci Environ Epidemiol 24, 537-544.

36. Yang X, Bao Y, Fu H, et al. (2014) Selenium protects neonates against neurotoxicity from prenatal exposure to manganese. PLOS ONE 9, e86611.

37. Anan Y, Ogra Y, Somekawa L, et al. (2009) Effects of chemical species of selenium on maternal transfer during pregnancy and lactation. Life Sci 84, 88-93. 
38. Darlow BA, Inder TE, Graham PJ, et al. (1995) The relationship of selenium status to respiratory outcome in the very low birth. Pediatrics 96, 314-319.

39. Taylor AE, Keevil B \& Huhtaniemi IT (2015) Mass spectrometry and immunoassay: how to measure steroid hormones today and tomorrow. Eur J Endocrinol 173, D1-D12.

40. Yang SJ, Hwang SY, Choi HY, et al. (2011) Serum selenoprotein $\mathrm{P}$ levels in patients with type 2 diabetes and prediabetes: implications for insulin resistance, inflammation, and atherosclerosis. J Clin Endocrinol Metab 96, E1325-E1329.
41. Ballihaut G, Kilpatrick LE, Kilpatrick EL, et al. (2012) Multiple forms of selenoprotein $\mathrm{P}$ in a candidate human plasma standard reference material. Metallomics 4, 533-538.

42. Akesson B, Bellew T \& Burk RF (1994) Purification of selenoprotein P from human plasma. Biochim Biophys Acta 1204, 243-249.

43. Hybsier S, Schulz T, Wu Z, et al. (2017) Sex-specific and inter-individual differences in biomarkers of selenium status identified by a calibrated ELISA for selenoprotein P. Redox Biol 11, 403-414. 